\title{
Facile green synthesis of graphene metal nanocomposites and their promising applications
}

\author{
Ashwini G. Patil ${ }^{1}$, Pravin O. Patil ${ }^{2, *}$, Mahesh P. More ${ }^{3}$, Prashant K. Deshmukh ${ }^{4}$, Sanchita S. Mahale ${ }^{5}$ \\ ${ }^{\mathbf{1}}$ Assistant Professor, ${ }^{2,4}$ Associate Professor, ${ }^{3,5}$ Student, ${ }^{1}$ Dept. of Microbiology, R. C. Patel ASC College, Shirpur, Dhule, \\ Maharashtra, ${ }^{2-5}$ Dept. of Quality Assurance, H. R. Patel Institute of Pharmaceutical Education and Research, Shirpur, Dhule, \\ Maharashtra, India
}

*Corresponding Author:

Email: rxpatilpravin@yahoo.co.in

\begin{abstract}
Graphene, a 2D carbon sheet, has attracted tremendous attention in the scientific fraternity. Recently, graphene based metal nanocomposites (GO@Me-NCs) have opened an exciting avenue amongst researchers worldwide, owing to its exceptional properties. Graphene has a high electrical conductivity, exceptional chemical tolerance and large surface-to-volume ratio, which makes it an obvious template for nanocomposites. As a result, incorporation of graphene with inorganic materials such as metal has been the spotlight of research in recent years for their multi-functionalities. From the perspective of chemistry and materials, this account presents an overview of the green and benign processes for fabrication of GO@Me-NCs and their wide range of applications in various fields. Present review highlights the fabrication of GO@Me-NCs by green techniques using plant extracts, carbohydrates, vitamins, amino acids, etc. Finally a concluding remarks are also provided for the researchers, who are interested in designing advanced green strategies for the fabrication of graphene based metal nanocomposites.
\end{abstract}

Keywords: Green synthesis, Graphene, Metal Nanocomposites.

\section{Introduction}

Recently the interests of scientists have explode growth in graphene, a single-atom-thick 2D layer of sp2-bonded carbon, have received tremendous attention owing to their unique and outstanding electronic, mechanical and thermal properties. ${ }^{1}$ Especially, graphene composites with metal or metal oxide nanoparticles and graphene itself, offer great potential for various applications such as catalysis, dye adsorption, electrical, optical, sensors and so on. ${ }^{2}$

Graphene oxide (GO), a chemically modified graphene contains a wide variety of reactive oxygenated functional groups such as hydroxyl, carboxylic and epoxides. ${ }^{3}$ As a result of the high reactivity and superior water dispersibility from the oxygenated groups, GO has been expressed as a promising materials to develop a novel graphene-based composite through chemical functionalization. ${ }^{4}$ Reduced graphene oxide (rGO) has been obtained from the reduction of GO using diverse chemical reducing agents. ${ }^{5}$ The chemical reducing agents designed for reduction of GO are highly toxic as well as hazardous. To surmount risk associated with chemical reducing agents and hydrothermal method, the use of nature based reducing agents and eco-friendly methods are eye-catching alternatives. Recently, excellent review demonstrating alternative methods and nature-based reagents for the reduction of GO is reported elsewhere. ${ }^{6}$

Metal nanoparticles (MNPs) promising role is rising in the technological revolution because of their unusual properties, that approach optics past the diffraction limit and fully into the nanometer size regime. $^{7}$
Amongst GO@Me-NCs, the noble metals such as silver $(\mathrm{Ag})$, gold $(\mathrm{Au})$, are the most emerged in literatures because of their catalytic and optical properties. ${ }^{8,9}$

Since the decade, many studies have been reported on the synthesis of these GO@Me-NCs. Amongst the various methods, the most common employs are the chemical reduction as well as hydrothermal process. In fact, excellent reviews have been published demonstrating the synthesis and applications of graphene based metal composites. ${ }^{10,11}$

However, to the best of our knowledge till date, no review appeared in literature on green methods for synthesis of GO@Me-NCs. Therefore, in the present review an innovative attempt has been made to highlight the fabrication of GO@Me-NCs using green techniques viz. plant extracts, carbohydrates, vitamins, amino acids, etc and their applications. Finally concluding remarks are also provided to the researchers, who are interested in designing advanced green strategies for the fabrication of GO@ Me-NCs.

\section{Fabrication and promising applications of GO@Me- NCs, synthesized using}

1. Plant extracts: Recently, the use of plant extracts, as one of the most promising natural reducing agents for the synthesis of GO@Me-NCs has attracted immense significance because plants are generally inexpensive, readily available and nontoxic. Moreover, it is advantageous over chemical and physical methods, as it is economic, single-step, high reaction rate, does not require high pressure, energy and temperature. A comprehensive investigation of plant extracts based 
green synthesis of GO@Me-NCs and their applications are discussed herein. Summary of synthesis of GO@Me-NCs using plant extracts are presented in Table 1.

2. Graphene silver nanocomposites: A facile green synthesis of graphene silver (GO-Ag) nanocomposite by using garlic extract and sunlight irradiation was reported. ${ }^{12}$ It was revealed that the garlic components used as a reducing and capping agents for the formation of silver nanoparticles (AgNPs). Synthesized nanocomposite modified with glassy carbon electrode was applied in electrochemical sensing of nitrite ions. Results demonstrated that GO-Ag nanocomposite can produce a synergistic catalytic current in nitrite oxidation with significant negative shift in over potential. The developed sensor was stable, reproducible and showed an excellent selectivity toward the detection nitrite ions among other common interferents. Additionally, it was also successfully applied for the detection of nitrite ions in lake water sample.

A simple ecofriendly technique for preparation of reduced graphene oxide silver (rGO-Ag) nanocomposite via the Tiliaamurensis plant extracts as a reducing as well as stabilizing agents is reported..$^{13}$ The fabricated $\mathrm{rGO}-\mathrm{Ag}$ nanocomposite exhibited significant cytotoxicity and showed highly effective apoptotic activity against ovarian cancer cells. It was demonstrated that $\mathrm{rGO}-\mathrm{Ag}$ nanocomposite yielded a synergistic effect over the bare nanomaterials. Therefore, this novel formulation may aid the development of better anticancer therapeutics and could provide new treatments for the cancer and cancer stem cells-targeted cancer therapies.

An eco-friendly synthesis of AgNPs and GOAgNPs through Picrasmaquassioides aqueous extract as a reducing and capping agents was accomplished. ${ }^{14}$ The resulted GO-AgNPs nanocomposites exhibited higher activity for degradation of methylene blue (MB) dye in the presence of sodium borohydride. With the use of GO-AgNPs catalysis of MB was achieved in 15 minutes only. RaziehMoosavi et al. demonstrated the facile and green method for the synthesis of metallic AgNPs and also fabricates GO, Ag NPs and chitosan [GO+Ag@CS] as well as GO, iron oxide and Ag NPs $[\mathrm{GO}+\mathrm{IO}+\mathrm{Ag}]$ nanocomposites via water-soluble plant extract of cinnamon as a green reducing and capping agent. ${ }^{15}$ The antibacterial effects of Ag NPs and [GO+IO+Ag] nanocomposites towards both Gramnegative bacteria E. coli and Gram-positive bacteria $\mathrm{S}$. aureuswere investigated. The nanocomposites $[\mathrm{GO}+\mathrm{IO}],[\mathrm{GO}+\mathrm{Ag}], \quad[\mathrm{GO}+\mathrm{Ag} @ \mathrm{CS}], \quad[\mathrm{GO}+\mathrm{IO}+\mathrm{Ag}$ NPs]) tested, the Ag NPs and[GO+Ag@CS], [GO+IO+Ag NPs] nanocomposites exhibited a lower MICof 0.025 against E. coli. On the contrary, these nanocomposites exhibited a higher MIC of 0.05 against S. aureus. An interesting finding was revealed about mechanism of antibacterial effect, the differences in the antimicrobial activity of the samples were owing to cell walls of the two types of bacteria.The cell wall of $E$. coli bacteria is made up of a thin peptidoglycan and lipopolysaccharide; later consist of covalently linked lipids and polysaccharides exhibiting a negative charge, which serves as a weak permeability barrier to the $\mathrm{Ag}$ NPs and the nanocomposites having a positive charge. In contrast, the cell wall of $\mathrm{S}$. aureus bacteria is made up of a thickpeptidoglycan layer comprising of linear polysaccharide chains which restricts the attachment or the penetration of the Ag NPs and the nanocomposites to the cell wall.

In situ green method for preparation of $\mathrm{rGO}-\mathrm{Ag}$ by using tea polyphenols (TPs) as both reducing and stabilizing agent is mentioned recently. ${ }^{16}$ The existence of epicatechin, epicatechin gallate, epigallocatechin and epigallocatechin gallate in TPs were held responsible for formation of $\mathrm{rGO}-\mathrm{Ag}$ nanocomposite. It was demonstrated that the uniform distribution of AgNPs, the high adsorption ability of graphene and the effective electron transfer from graphene to AgNPs afforded the rGO-Ag nanohybrid as an efficient catalyst in the reduction of 4-nitrophenol (4-NP).This green and novel synthesis route provided a useful platform for the fabrication of GO@Me-NCs heterogeneous catalyst, which might be able to find wide spread use in a number of practical catalytic applications. The synthesis of Ag-rGO nanocomposite was attempted using an aqueous extract of Colocasia esculenta leaf as reducing agent. ${ }^{17}$ The polyphenolic compounds present in the extract facilated the reduction of $\mathrm{Ag}+$ ions to $\mathrm{Ag} 0$ ions. This Ag-rGO composite demonstrated deposition of the spherical shapes silver nanoparticles over the surface of rGO sheets. Due to excellent cytocompatibility against peripheral blood mononuclear cells and mammalian red blood cells, this composite showed better antimicrobial activity than nanoparticles alone. Moreover, it is worthy to mention that because of acute dermal toxicity study on wistar rats, this nanocomposite also applied as a topical antimicrobial agent for dressing, bandages and ointments etc.

A simple, environmentally friendly and low-cost method for the reduction of graphene oxide and preparation of reduced $\mathrm{rGO} /$ mono and bimetallic nanocomposites via Azadirachtaindica extract is reported. ${ }^{18}$ The presence of alkaloids, flavonoids and terpenes were found to be responsible for reduction as well as synthesis of mono and bimetallic nanocomposites. The fabricated $\mathrm{Ag}-\mathrm{Au}$ bimetallic nanoparticle decorated rGO nanocomposite was successfully utilized for non-enzymatic amperometric sensing of hydrogen peroxide with excellent selectivity, a wide linear range, rapid response, stability and a lower detection limit. Therefore the biomedical approach permits a promising platform for the application of rGO based metal nanocomposites in the non-enzymatic amperometric sensor field. Green and 
one step method for the simultaneous reduction of silver ion and GO by Pulicariaglutinosa plant extract in the formation of highly reduced graphene oxide/Ag (PE-HRG-Ag) nanocomposites is also performed. ${ }^{19}$ The Pulicariaglutinosaextract have reduction as well as stabilizing activity for silver ions on the surface of GO, due to its flavonoids and polyphenols contents. The prepared PE-HRG-Ag nanocomposites display excellent surface-enhanced Raman scattering (SERS) activity and significantly increased the intensities of the Raman signal of graphene for the detection of chemical and biological analytes.

Sedki and co-workers utilized PotamogetonPectinatus plant extract as a reducing agent to synthesize the silver-reduced graphene oxide (Ag-rGO) nanocomposite. ${ }^{20}$ This plant extract is rich with polyphenolic compounds, proteins, amino acids and carbohydrate, which possesses a reducing as well as stabilizing activity for nanocomposite synthesis. Note worthily the polyphenolic compounds in particular flavonoids chelated metal ion and then reduced them by donation of electron or hydrogen atoms. Alternatively, the protein with amine and carboxylate groups acts as reducing agent while a capping and stabilizing agent due to carbonyl groups which adhere to metal nanoparticles. The Ag-rGO nanocomposite demonstrated more potent antibacterial activity against E. coli bacteria than GO nanosheets due to synergistic effect of both AgNPs and rGO nanosheets.

\section{Graphene gold nanocomposites}

Synthesis of rGO decorated with gold ( $\mathrm{rGO}-\mathrm{Au}$ ) nanoparticles using rose water as reducing agent was performed by green approach. ${ }^{21}$ Wherein, the direct electrochemistry of glucose oxidase (GOD) was achieved at a glassy carbon electrode. The resulting biosensor exhibited a good response to glucose with a linear range from 1 to $8 \mathrm{mM}$ with a low detection limit of $10 \mu \mathrm{M}$. Results revealed that the prepared modified electrode with $\mathrm{rGO}-\mathrm{Au}$ nano nanocomposite would enhance the direct electron transfer capability of GOD on the electrode surfaces. The fabricated biosensor exhibited high sensitivity, anti-fouling properties, the lower detection limit and high stability for glucose sensing. An interesting finding about cost effective and ecofriendly use of wastes of willow bark as a carbon source for hydrothermal preparation of water soluble, photo luminescent carbon dots (CDs) has been reported. $^{22}$ The synthesized CDs were used as an effective photo catalyst for fabricating AuNPs-rGO nanocomposites in the presence of UV light. The fabricated AuNPs-rGO nanocomposite was successfully utilized for the catalytic performance of hydrogen peroxide reduction and oxidation. In addition, fabricated nanocomposites were also used for glucose detection by immobilizing GOD on the AuNPs-rGO modified glassy carbon electrode (GOX-AuNPs-rGOGCE).

A multifunctional and multisynergistic hydrogel system containing rGO, Amaranth extract and AuNPs (rGO-AE-AuNPs) has been synthesized by Chang et al., for high-performance antitumor therapy. ${ }^{23}$ In this approach for the first time Amaranth extract has been used directly as a low toxicity and ecofriendly photosensitizer for antitumor effect, a cross-linking agent for the formation of rGO-based hydrogel and a green reductant for the preparation of AuNPs. Prepared hydrogel showed features like simple preparation process, low cost and good biocompatibility. The composite hydrogel was remarkably improved and synergistic antitumor effects and could serve as photodynamic therapy (PDTs)/photothermal therapy (PTT) unified platform cancer treatment through sequential irradiation as stimuli. Simsikovaetal, described a green, efficient and simple approach for the synthesis of gold flowerlike structures by assembling AuNPs on rGO surface in the presence of green tea. ${ }^{24}$ Here in, catalytic activity of nanoflower in degradation of organic dyes (Congo red, safranin T, and eosin Y) has been described. Utilizing synergistic advantages of chitosan, AuNPs and graphene nanosheets, a new plant esterase-based (PLaE-CS-AuNPs-GNs) biosensor has been synthesized to achieve ultrasensitive and selective detection of organophosphorus pesticides spiked in carrots and apples. ${ }^{25}$ Plant esterase (PLaE), was purified from plants and then used as an alternative for expensive AChE without sacrifice of the biosensing performance also for ultrasensitive and selective detection of organophosphate pesticides in carrot and apple.

Table 1: GO@MNCs using plant extracts

\begin{tabular}{|c|l|l|l|c|}
\hline S. No. & \multicolumn{1}{|c|}{ GO@MNC } & Source / Method & Applications & Reference \\
\hline 1 & GO-Ag & $\begin{array}{l}\text { Fresh garlic (Allium } \\
\text { sativum) cloves } \\
\text { extract and sunlight }\end{array}$ & $\begin{array}{l}\text { Electrochemical detection of } \\
\text { nitrite ions. }\end{array}$ & 12 \\
\hline 2 & rGO-Ag & $\begin{array}{l}\text { Tiliaamurensis plant } \\
\text { extracts }\end{array}$ & Anticancer nanotherapy & 13 \\
\hline 3 & GO-AgNPs & $\begin{array}{l}\text { Picrasmaquassioides } \\
\text { bark aqueous extract }\end{array}$ & Degradation of methylene blue & 14 \\
\hline 4 & GO + Ag-CS and & Plant extract of & Antibacterial & 15 \\
\hline
\end{tabular}




\begin{tabular}{|c|c|c|c|c|}
\hline & $\mathrm{GO}+\mathrm{IO}+\mathrm{Ag}$ & cinnamon & & \\
\hline 5 & Ag-TPG & Tea polyphenols & $\begin{array}{l}\text { Catalytic reduction of 4- } \\
\text { nitrophenol }\end{array}$ & 16 \\
\hline 6 & Ag-rGO & $\begin{array}{l}\text { Colocasiaesculentale } \\
\text { af aqueous extract }\end{array}$ & $\begin{array}{l}\text { Topical antimicrobial agent in } \\
\text { dressing, bandage, ointments } \\
\text { etc. }\end{array}$ & 17 \\
\hline 7 & $\begin{array}{l}\mathrm{Ag}-\mathrm{rGO} \text { and } \\
\mathrm{Ag}-\mathrm{Au}-\mathrm{rGO}\end{array}$ & $\begin{array}{l}\text { Azadirachtaindica } \\
\text { leaves extract }\end{array}$ & Non-enzymatic $\mathrm{H}_{2} \mathrm{O}_{2}$ sensing. & 18 \\
\hline 8 & PE-HRG-Ag & $\begin{array}{l}\text { Whole } \\
\text { Pulicariaglutinosa } \\
\text { plant extract }\end{array}$ & SERS substrate & 19 \\
\hline 9 & Ag-rGO & $\begin{array}{l}\text { Potamogetonpectinat } \\
\text { us plant aqueous } \\
\text { extract }\end{array}$ & Antibacterial & 20 \\
\hline 10 & rGO-Au & Rose Water & Glucose biosensing & 21 \\
\hline 11 & AuNPs-rGO & Willow bark waste & $\begin{array}{l}\text { Catalytic performance for } \\
\mathrm{H}_{2} \mathrm{O}_{2} \text { reduction and for } \\
\text { glucose biosensing }\end{array}$ & 22 \\
\hline 12 & rGO-AE- AuNPs & $\begin{array}{l}\text { Amaranth leaves } \\
\text { extract }\end{array}$ & Antitumor & 23 \\
\hline 13 & rGO-AuNPs & Green tea & $\begin{array}{l}\text { Degradation of organic dyes } \\
\text { (Congo red, safranin } \mathrm{T} \text {, and } \\
\text { eosin Y) }\end{array}$ & 24 \\
\hline 14 & $\begin{array}{l}\text { PLaE-CS-AuNPs- } \\
\text { GNs }\end{array}$ & Plant esterase & $\begin{array}{l}\text { Detection of } \\
\text { organophosphorus pesticides }\end{array}$ & 25 \\
\hline
\end{tabular}

\section{Vitamins}

Vitamin C (L-Ascorbic acid) is a well-known natural reducing agent or antioxidant essential for many metabolic functions in living organisms. Due to such advantages it has been widely used as an ideal nontoxic substitute for toxic reductants e.g. hydrazine and sodium borohydride. In recent reports it is demonstrated that Vitamin $\mathrm{C}$ could reduce the oxygen containing groups, such as carboxylic and epoxy on the surface of GO. This product of the reduction is comparatively stable and uncreative which help to avoid agglomeration. Summary of synthesis of GO@Me-NCs using vitamins $C$ is presented in Table 2 .

\section{Graphene silver nanocomposites}

Hui et al. reported an ecofriendly synthesis of $\mathrm{Ag}$ nanoparticle-decorated graphene oxide (AgNP-GO) composites by in-situ ultrasonication assistance at room temperature by means of Vitamin $\mathrm{C}$ as reducing agent. ${ }^{26}$ It was proposed that the positively charge $\mathrm{Ag}$ ions attracted to negatively charge oxygen containing functional group on GO surface by electrostatic force and deposited on GO surface because of $\mathrm{Ag}^{+}-\pi^{*}$ orbital interaction. This nanocomposite may hold a promise as advanced materials for various analytical applications such as catalysis, sensors and microchips. Synthesis of $\mathrm{Ag}-\mathrm{Co} / \mathrm{rGO}$ nanocomposite at room temperature with assistance of sodium borohydride $\left(\mathrm{NaBH}_{4}\right)$ for catalysis of 4-nitrophenol is reported with the assistance of LVitamin C. ${ }^{27}$ High catalytic activity of $\mathrm{Ag}-\mathrm{Co} / \mathrm{rGo}$ nanocomposite was due to a high conductivity of $\mathrm{Ag}$ which do not affect the borate ions, secondly the high rate generation of hydrogen on nanocatalyst co surface and finally the contact of 4-nitrophenol on surface of Ag. Additionally, rGO gives support for the 4nitrophenol molecule and assists in the easy hydrogen transfer to $\mathrm{sp}^{2}$ hybridized nitro group via own $\pi-\pi$ interactions.

Synthesis of AgNPs supported on nitrogen doped graphene (AgNPs-N-G) hybrid material was accomplished using Vitamin C. ${ }^{28}$ It was confirmed that the addition of nitrogen species onto the graphene surface using urea as $\mathrm{N}$-dopant was advantageous for AgNPs deposition. The $\mathrm{N}$ groups on $\mathrm{N}-\mathrm{G}$ served as nucleation and anchor sites for the deposition of $\mathrm{Ag}$ nanocrystals. Furthermore, the $\mathrm{N}$ atom has a lone pair of electron and $\mathrm{Ag}$ with unoccupied d-orbitals, as a result $\mathrm{N}$ atom facilitated to coordinate with the $\mathrm{Ag}$ nanocrystals which resulted in prevention of AgNPs agglomeration. Results revealed that AgNPs-N-G has a lower electrical resistance and higher electrocatalytic activity compared to undoped counterparts (AgNPsrGO) via electrochemical measurement and therefore it was effectively used for a nonenzymatic $\mathrm{H}_{2} \mathrm{O}_{2}$ sensing. Reddy et al. has been prepared AgI nanoparticle functionalized self assembledrGO aerogels by using vitamin $\mathrm{C}$ as the reducing agent. ${ }^{29}$ AgI-rGO aerogels exhibited high photocatalytic degradation ability to an organic dye (Rhodamine-B) due to high visible light driven catalytic activity of $\mathrm{AgI}$ and the high specific surface area of graphene nanosheets with 3D interconnected pores. In addition resultant hybrid 
material also applied for the reduction of 4-nitrophenol and synthesis of bis (indolyl) methane due to efficient charge separation and transportation between rGO and the AgI nanostructure. The formation rGO based $\mathrm{Ag}$ nanoparticles containing nanocomposite hydrogel by in-situ reduction of $\mathrm{GO}$ using vitamin $\mathrm{C}$ with polyethyleneimine (PEI) was attained. ${ }^{30}$ Wherein, construction of $\mathrm{rGO} / \mathrm{PEI} / \mathrm{Ag}$ hydrogels demonstrated excellent photocatalytic activity in Rhodamine-B and methylene blue. Consequently, this nanocomposite was utilized in the waste water treatment as an exceptionally efficient dye catalyst.

Kavinkumar and Manivannan reported the preparation AgNP-GO nano sheets using vitamin C. ${ }^{31}$ The fabricated AgNP-GO efficiently utilized for ammonia sensing in the concentration ranging from 0 to $500 \mathrm{ppm}$ which could results in to selective ammonia sensing for environmental and industrial applications. Jian Liu et al. developed a green approach for the synthesis of rGO films and further these films were fabricated with the AgNPs to formed rGO-Ag nanocomposite by using vitamin $\mathrm{C} /$ water vapor as the reducing agent. ${ }^{32}$ Except for the use of gaseous hydrazine hydrate or hydroiodic acid, this was the first reported use of an environmentally friendly gaseous phase for the reduction of graphene oxide into $\mathrm{rGO}$ films. The vitamin $\mathrm{C}$ /water vapor were able to penetrate into the GO films and reduce the Ag ions. The
rGO-Ag composite films effectively used as SERS substrates and also showed antibacterial activity against Escherichia coli, Staphylococcus aureus. The synthesized $\mathrm{rGO}-\mathrm{Ag}$ composites have great potential for use in the detection of small molecules and as selective antibacterial nanomaterials.

\section{Graphene gold nanocomposites}

Maria Iliut and coworker reported a novel approach for the synthesis of aqueous stable rGO-AuNP hybrids using polyvinyl pyrolidone (PVP) as stabilizer and vitamin $\mathrm{C}$ as reducing agents. ${ }^{33}$ Authors had investigated the influence of temperature and concentration of metal precursors on the size of AuNPs. The fabricated hybrids were successfully utilized as the SERS substrate for the detection of Nile Blue A in a liquid environment and their performance was increase with AuNP density on the rGO surface. Recently a novel, green and easy method was reported for the synthesis of rGO-MB-AuNPs composites using simultaneous vitamin $\mathrm{C}$ as the main and methylene blue (MB) the auxiliary reductant. ${ }^{34}$ Owing to novel structure and the combination of excellent properties of $\mathrm{MB}, \mathrm{rGO}$, and AuNPs the synthesized rGO-MB-AuNPs composites have a practical application in electrochemical analysis. The composites exhibited an excellent response to the oxidation of uric acid and dopamine.

Table 2: Synthesis of GO@MNCs using Vitamins

\begin{tabular}{|c|l|c|l|c|}
\hline S. no. & \multicolumn{1}{|c|}{ GO@ MNC } & Source/method & \multicolumn{1}{|c|}{ Application } & Reference \\
\hline 1 & AgNP-GO & Vitamin C & $\begin{array}{l}\text { Analytical applications such as } \\
\text { catalysis, sensors and microchips }\end{array}$ & 26 \\
\hline 2 & Ag-Co-rGO & Vitamin C & $\begin{array}{l}\text { Catalytic reduction of 4- } \\
\text { nitrophenol }\end{array}$ & 27 \\
\hline 3 & AgNPs-N-G & Vitamin C & Nonenzymatic sensing of $\mathrm{H}_{2} \mathrm{O}_{2}$ & 28 \\
\hline 4 & AgI-rGO & Vitamin C & $\begin{array}{l}\text { Degradation of Rhodamine B, } \\
\text { reduction of 4-NP, and synthesis } \\
\text { of bis(indolyl)methane }\end{array}$ & 29 \\
\hline 5 & rGO-PEI-Ag & Vitamin C & $\begin{array}{l}\text { Degradation of Rhodamine-B and } \\
\text { methylene blue }\end{array}$ & 30 \\
\hline 6 & AgNP-GO & Vitamin C & Ammonia sensing & 31 \\
\hline 7 & rGO-Ag & $\begin{array}{c}\text { Vitamin C /water } \\
\text { vapor }\end{array}$ & SERS substrate and antibacterial & 32 \\
\hline 8 & rGO-AuNP & Vitamin C & $\begin{array}{l}\text { SERS substrate detection of Nile } \\
\text { blue }\end{array}$ & 33 \\
\hline 9 & rGO-MB-AuNPs & Vitamin C & Detection of uric acid dopamine & 34 \\
\hline
\end{tabular}

\section{Amino acids \\ Graphene silver nanocomposites}

Amino acids are the building blocks of proteins and have held huge potential for synthesis of graphene based nanomaterials. It has been well - documented that some amino acids (e.g., Cysteine, lysine, arginine and tryptophan) may serve as good reducing as well capping agents for the synthesis of GO@MNCs.
Summary of synthesis of GO@Me-NCs using amino acids are presented in Table 3.

A facile and rapid microwave assisted preparation of Ag-rGO NCs via simultaneous reduction of AgNPs and GO by L-arginine is recently reported..$^{35}$ The AgrGO nanocomposites were demonstrated to be suitable SERS substrates with high sensitivity and outstanding uniformity owing to the detection of the common Raman reporter molecules, 4-aminothiophenol. 
On similar line same research group have synthesized Ag-rGO nanocomposite using L-arginine. ${ }^{35}$ Where, an excellent catalytic activity and stability for the reduction of 4-nitrophenol to 4-aminophenol with sodium borohydride was achieved using Ag-rGO. The catalysis obeyed the pseudo-first-order kinetics and its rate constant is depends not only on temperature and catalyst amount but also concentration of 4nitrophenol.In-situ water dispersible Ag-GO nanocomposite were synthesised using tryptophan, which also acts as a stabilizing agent for the NCs. ${ }^{36}$ This NCs exhibited outstanding SERS activity like SERS alternative and it is used for detection of crystal violet in water.

\section{Graphene gold nanocomposites}

Green and environment friendly in situ fabrication of graphene oxide/gold nanoparticle (GO-AuNPs) hybrid is reported by Wen Liang $\mathrm{Fu}$ and co-worker using tyrosine as biocompatible reducing agent. ${ }^{37}$ The fabricated GO-AuNPs hybrid was effectively utilized for label-free malachite green (MG) detection in water.
The synthesized GO-AuNPs hybrids can also be used as efficient SERS substrates. The GO exhibited a strong enrichment effect on $\mathrm{MG}$ due to the electrostatic interaction and $\pi-\pi$ stacking, and the in situ synthesized GO-AuNPs hybrids greatly enhanced the Raman scattering signal of $\mathrm{MG}$ which was resulted in high sensitivity.

A new sandwich-type electrochemical luminescence (ECL) immune sensor for the detection of CA199 in real serum samples based on a $\mathrm{GN}-\mathrm{Ag}-\mathrm{Au}$ as sensor platform and GQDs functionalized PtPd nanochains for signal amplification is designed by Yang and co-workers, recently. ${ }^{36}$ The $\mathrm{GN}-\mathrm{Ag}-\mathrm{Au}$ as a sensor platform was executed by chemical reduction method and was used to transform the electrode, increasing the surface area to capture a large amount of primary antibodies as well as improving the electronic transmission rate. The proposed ECL immunosensor exhibited good precision, acceptable stability and reproducibility, and could be used for the detection of CA199 in real serum samples.

Table 3: Synthesis of GO@MNCs using amino acids ac-37 $^{35}$

\begin{tabular}{|c|l|c|l|c|}
\hline S. No. & GO@MNC & $\begin{array}{c}\text { Amino acid } \\
\text { used }\end{array}$ & Application & Reference \\
\hline 1 & Ag-rGO & L-arginine & SERS substrate & 35 \\
\hline 2 & Ag-rGO & L-arginine & $\begin{array}{l}\text { Catalytic reduction of 4- } \\
\text { nitrophenol }\end{array}$ & 35 \\
\hline 3 & Ag-GO & Tryptophan & Crystal violet detection in water & 36 \\
\hline 4 & GO-AuNPs & Tyrosine & $\begin{array}{l}\text { Label-free malachite green } \\
\text { (MG) detection in water and as } \\
\text { efficient SERS substrates }\end{array}$ & 37 \\
\hline
\end{tabular}

\section{Carbohydrates}

Carbohydrates (biopolymers) are another family of natural sources used as reducing and stabilizing agents for synthesis of GO@Me-NCs. These polymeric carbohydrate molecules have already been used in a variety of industries and therefore are readily available for the large-scale production of graphene basednanomaterials. Examples of biopolymers for GO@Me-NCs synthesis are glucose, cyclodextrin, chitosan and polysaccharides. Summary of synthesis of GO@Me-NCs using carbohydrates are presented in Table 6.

\section{Graphene silver nanocomposites}

Synthesis of AgNPs deposited on rGO (rGOAgNPs) under microwave irradiation was reported by Yujie Han and co-workers using starch as the reducing as well as stabilizing agent. ${ }^{38}$ Synthesized rGO-AgNPs nanocomposite exhibited higher antimicrobial activity against gram negative bacteria Pseudomonous aeruginosa than bare AgNPs. Owing to cost effectiveness this NCs may be used as an effective antimicrobial agent in the industry. Li et al. developed an AgNPs-GO nanocomposite through a green and low- cost method by using glucose as a reductant and stabilizer. ${ }^{39}$ Glucose is a common natural reducing sugar and has been used as reducing agents due to their mild reductive ability and nontoxic property. This AgNPs-GO nanocomposite showed excellent repeatability and long-term stability, which may be applied as a sensor for determination of tryptophan in human fluids and pharmaceutical preparations. This developed nanocomposite sensor was successfully applied for the selective determination of tryptophan in the presence of a high concentration of tyrosine in human fluids and pharmaceutical preparations.

In contrast, AgNPs decorated on GO (GO-Ag) in super critical carbon dioxide $\left(\mathrm{scCO}_{2}\right)$ using a glucose as a reducing agent was established by Haldoraiet al. ${ }^{40}$ The $\mathrm{scCO}_{2}$ is generally used supercritical fluids (SCF) because of non-toxic, non-flammable and chemically inert and therefore used for fabrication of nanocomposite. It was revealed that the existence of carboxyl groups in GO may support the adsorption of $\mathrm{Ag}$ ions and stabilization of the newly produced AgNPs. As a result, the carboxyl groups in GO may work equally as a reaction center and stabilizer and the reduction was carried out on the planar surface of GO. 
The decorated nanocomposite not only demonstrated an excellent visible-light photocatalytic activity in degradation of Rhodamine 123 dye and acetaldehyde but also exceptional antibacterial activity against pathogens.

Synthesis and antibacterial activity of GO-Ag nanocomposite was accomplished using glucose as a reducing and starch as stabilizing agent. ${ }^{23}$ It was concluded that the GO surface play a vital role in the process of nucleation and stabilization for formation of AgNPs and starch as a stabilizer to prevent the agglomeration of AgNPs. The nanocomposite showed excellent antibacterial activity and low cell cytotoxicity against E. coli and $S$. aureus.

Jinchen Fan et al. reported the graphene-Ag (G$\mathrm{Ag}$ ) nanohybrids by using gum arabic as reducing and stabilizing agent. ${ }^{41}$ This synthesized G-Ag nanohybrid is a suitable alternative to SERS for the detection of 4aminothiophenol in a liquid medium. Fabrication of silver nanoparticle anchored on graphene surface (AgNPs- $\beta$-CD-Gr) by in-situ thermal reduction of GO and $\mathrm{AgNO}_{3}$ through $\beta$-cyclodextrin $(\beta-\mathrm{CD})$ as a reductant and stabilizer was accomplished by Huiet $a l{ }^{42}$ The $\beta$-CD consists of six $\alpha$-cyclodextrin, seven $\beta$ cyclodextrin, eight $\gamma$-cyclodextrin or more glucopyranose units linked by $\alpha-(1,4)$ bonds is not only stabilizing and dispersing ability for nanoparticle but also have reduction ability to formed nanoparticle on supporting materials. TheAgNPs- $\beta-C D-G r$ nanocomposite assembled with gassy carbon electrode (GCE) to form AgNPs- $\beta-C D-G r-G C E$ and applied for simultaneous determination of guanine and adenine by differential pulse voltammograms. Moreover, this biosensor was also applied for detection of trace levels of these purine bases in herring sperm DNA as electrocatalytic biosensing.

Synthesis of Ag-rGO nanocomposites were achieved using chitosan as a reducing agent (Chen and groups). ${ }^{43}$ The functionalization of the synthesized AgNC-rGO nanocomposite was accomplished using a poly (5-amino-1, 3, 4-thiadiazole-2-thiol) (p-ATT) modified carbon fiber disk ultramicroelectrode (CFME) for the instantaneous detection of hydroquinone (HQ) and catechol (CT). Results revealed that the AgNCsrGO-p-ATT-CFME displayed a low detection limit, representing it might be used as a potential sensing platform for the detection of HQ and CT. Chook et al. synthesized silver nanoparticles and graphene oxide nanocomposite by in-situ synthesis with the addition of chitosan which can serve as a binding agent. ${ }^{44}$ This formed nanocomposite used for antibacterial purpose and could be potentially used in food packaging and biomedical applications.

Indranil Roy and co-workers mentioned the use of lactulose as a reducing and stabilizing agent in the preparation of NCs based on reduced graphene oxide and silver nanoparticles (Ag-rGO). ${ }^{45}$

\section{Graphene gold nanocomposites}

New benign method for synthesis of gold nanoparticle functionalized graphene $(\mathrm{Au}-\mathrm{Gr})$ from $\beta$ cyclodextrin functionalized $\mathrm{Gr}$ (CD $\mathrm{Gr}$ ) was attempted. ${ }^{46}$ The attempted functionalized nanohybrid was used for electrochemical sensing of glucose and vitamin C. This excellent electrochemical sensing was due to the synergistic effect of Au NPs and excellent properties of graphene.

Table 4: Synthesis of GO@MNCs using carbohydrates ${ }^{23,38-46}$

\begin{tabular}{|c|l|c|l|c|}
\hline S. no. & GO@MNC & Source/method & \multicolumn{1}{|c|}{ Application } & Reference \\
\hline 1 & rGO-AgNPs & Starch $(\mathrm{MW})$ & Antimicrobial & 38 \\
\hline 2 & AgNPs-GO & Glucose & $\begin{array}{l}\text { Electrochemical sensing of } \\
\text { tryptophan }\end{array}$ & 39 \\
\hline 3 & GO-Ag & Glucose & $\begin{array}{l}\text { Degradation of organic } \\
\text { pollutants }\end{array}$ & 40 \\
\hline 4 & GO-Ag & Glucose & Antibacterial & 23 \\
\hline 5 & Ag-GA-G & Gum arabic & $\begin{array}{l}\text { SERS substrate for the } \\
\text { detection of 4- } \\
\text { aminothiophenol (4-ATP) }\end{array}$ & 41 \\
\hline 6 & $\begin{array}{l}\text { AgNPs- } \beta-C D- \\
\text { Gr }\end{array}$ & $\begin{array}{l}\beta-\text { cyclodextrin } \\
(\beta-C D)\end{array}$ & $\begin{array}{l}\text { Electrocatalytic biosensing of } \\
\text { purine bases }\end{array}$ & 42 \\
\hline 7 & AgNC-rGO & Chitosan & $\begin{array}{l}\text { Simultaneous determination of } \\
\text { hydroquinone and } \\
\text { catechol }\end{array}$ & 43 \\
\hline 8 & Ag-GO & Chitosan & Antibacterial & 44 \\
\hline 9 & RGO-Ag & Lactulose & - & 45 \\
\hline 10 & Au-Gr & Cyclodextrine & $\begin{array}{l}\text { Electrochemical sensing of } \\
\text { glucose and vitamin C }\end{array}$ & 46 \\
\hline
\end{tabular}




\section{Concluding remarks}

Green synthesis of graphene based metal nanomaterial, may hold the key to building an environmentally sustainable society in the twenty-first century. In the plant extracts based GO@ Me-NCs, the rate of the reaction is moderately high and the reaction takes several minutes to several hours to complete, depending on the plant type and amount. Plant extractmediated synthesis of graphene metal nanomaterials mainly occurs at room temperature. For these reasons, plant extracts are more appropriate for large-scale production. The exploitation of living plants may be promising as a cost- effective loom for the synthesis of GO@ Me-NCs.

Analogous to other natural reducing agents, vitamin $C$ could be effectively utilized for the synthesis of GO@ Me-NCs. Regardless of the widespread commercial availability of vitamin $\mathrm{C}$; its cost effectiveness may be an obstacle for the commercial synthesis of GO@Me-NCs.

In addition, amino acids alone may not be adequate for the controlled synthesis of GO@ Me-NCs. The adjustment of macro environmental factors (e.g., temperature, $\mathrm{pH}$ etc) undoubtedly has their practical limits, and this has direct to an interest in developing a different approach. Therefore, proteins and peptides also suffer from cost-related challenges and thus are illsuited for the large-scale production of GO@ Me-NCs. These relevant green methods for the fabrication of GO@ Me-NCs could provide an effective boost to the development and implementation of green nanotechnologies on a commercial scale production of GO@Me-NCs.

\section{Acknowledgements}

The authors are also grateful to the Management and Principal, R. C. Patel, ASC, College and H. R. Patel Institute of Pharmaceutical Education and Research, Shirpur for providing the necessary facilities, support and encouragement.

\section{References}

1. Geim, A. K. and NovoselovK. S. (2007). Nature materials 6(3):183-91.

2. Li, D., MuellerM. B., GiljeS., KanerR. B. and Wallace G. G. (2008). Nature nanotechnology 3(2): 101-105.

3. Allen, M. J.,TungV. C. and KanerR. B. (2009). Chemical reviews 110(1):132-45.

4. Pasricha, R., Gupta S. and SrivastavaA. K. (2009). Small 5(20): 2253-2259.

5. Stankovich, S., DikinD. A., PinerR. D., KohlhaasK. A., KleinhammesA., JiaY., WuY., Nguyen S. T. and Ruoff R. S. (2007). Carbon 45(7):1558-65.

6. Thakur, S. and KarakN. (2015). Carbon 94:224-42.

7. Lal, S., Link S. and Halas N. J. (2007). Nature photonics 1(11):641-8.

8. Cui, J. and Liu Y. (2015). RSC Advances 5(104):85748-55.

9. Turcheniuk, K., BoukherroubR. and SzuneritsS. (2015). Journal of Materials Chemistry B 3(21):4301-24.

10. Xu, C., Wang X. and Zhu J. (2008). The Journal of Physical Chemistry C 112(50):19841-5.
11. Khan, M., TahirM. N., AdilS. F., KhanH. U., SiddiquiM. R. H., Al-warthanA. A. and TremelW. (2015). Journal of Materials Chemistry A 3(37):18753-808.

12. Ikhsan, N. I., RameshkumarP., PandikumarA., ShahidM. M., HuangN. M., Kumar S. V. and Lim H. N. (2015). Talanta 144:908-14.

13. Gurunathan, S., HanJ. W., ParkJ. H., KimE., ChoiY.-J., Kwon D.-N. and Kim J.-H. (2015). International journal of nanomedicine 10:6257.

14. Sreekanth, T., Jung M.-J. andEomI.-Y. (2016). Applied Surface Science 361:102-6.

15. Moosavi, R., RamanathanS., LeeY. Y., LingK. C. S., AfkhamiA., ArchunanG., PadmanabhanP., GulyásB., KakranM. and SelvanS. T. (2015). RSC Advances 5(93):76442-50.

16. Wang, Z., XuC., Li X. and Liu Z. (2015). Colloids and Surfaces A: Physicochemical and Engineering Aspects 485:102-10.

17. Barua, S., ThakurS., AidewL., BuragohainA. K., ChattopadhyayP.andKarakN. (2014). RSC Advances 4(19):9777-83.

18. Babu, K. J., NahmK. S. and Hwang Y. J. (2014). RSC Advances 4(16):7944-51.

19. Al-Marri, A. H., KhanM., KhanM., AdilS. F., AlWarthanA., AlkhathlanH. Z., TremelW., LabisJ. P., SiddiquiM. R. H. and Tahir.M. N., 2015. International journal of molecular sciences 16(1), 1131-42.

20. Sedki, M., MohamedM. B., FawzyM., AbdelrehimD. A. and Abdel-MottalebM. M. (2015). RSC Advances 5(22):1735865.

21. Tabrizi, M. A. and VarkaniJ. N. (2014). Sensors and Actuators B: Chemical 202:475-82.

22. Qin, X., LuW., AsiriA. M., Al-YoubiA. O. and Sun X. (2013). Catalysis Science \& Technology 3(4):1027-35.

23. Chang, G., WangY., GongB., XiaoY., ChenY., WangS., LiS., HuangF., Shen Y.andXieA. (2015). ACS applied materials \& interfaces 7(21):11246-56.

24. Šimšíková, M., BartošM., ČechalJ. and ŠikolaT. (2016).Catalysis Science \& Technology.

25. Bao, J., HouC., ChenM., LiJ., HuoD., YangM., LuoX.and Lei Y. (2015). Journal of agricultural and food chemistry 63(47):10319-26.

26. Hui, K., HuiK., DinhD., TsangC., ChoY., ZhouW., Hong X. and Chun H.-H. (2014). ActaMaterialia 64:326-32.

27. Krishna, R., FernandesD. M., DiasC., VenturaJ., RamanaE. V., FreireC. and Titus E. (2015). International Journal of Hydrogen Energy 40(14):4996-5005.

28. Tian, Y., WangF., LiuY., Pang F. and Zhang X. (2014).ElectrochimicaActa 146:646-53.

29. Reddy, D. A., ChoiJ., LeeS., Ma R. and Kim T. K. (2015). RSC Advances 5(83):67394-404.

30. Jiao, T., GuoH., ZhangQ., PengQ., TangY., Yan X. and Li B. (2015). Scientific reports 5.

31. Kavinkumar, T. and ManivannanS. (2016). Ceramics International 42(1):1769-76.

32. Liu, J., LiuL., WuX., Zhang X. and Li T. (2015). New Journal of Chemistry 39(7):5272-81.

33. Iliut, M., LeordeanC., CanpeanV., Teodorescu C.M.andAstileanS. (2013). Journal of Materials Chemistry C 1(26):4094-104.

34. Zhang, Z., SunT., ChenC., XiaoF., Gong Z. and Wang S. (2014). ACS applied materials \& interfaces 6(23): 2103540.

35. Hsu, K.-C.and Chen D.-H. (2014). Nanoscale research letters 9(1):1-10.

36. Yang, B., LiuZ., GuoZ., ZhangW., WanM., Qin X. and ZhongH. (2014).Applied Surface Science 316:22-7. 
37. Fu, W. L., Zhen S. J. and Huang C. Z. (2013). Analyst 138(10):3075-81.

38. Han, Y., LuoZ., YuwenL., TianJ., Zhu X. and Wang L. (2013).Applied Surface Science 266:188-93.

39. Li, J., KuangD., FengY., ZhangF., XuZ., Liu M. and Wang D. (2013). Biosensors and Bioelectronics 42:198-206.

40. Haldorai, Y., KimB.-K., Jo Y.-L. and Shim J.-J. (2014). Materials Chemistry and Physics 143(3):1452-61.

41. Fan, J., ShiZ., GeY., WangJ., Wang Y. and Yin J. (2012). Journal of Materials Chemistry 22(27):13764-72.

42. Hui, Y., MaX., HouX., Chen F. and Yu J. (2015). Ionics 21(6):1751-9.

43. Chen, R., WangQ, LiY., GuY., TangL., Li C.and Zhang Z. (2015). RSC Advances 5(55):44165-72.

44. Chook, S. W., ChiaC. H., ZakariaS., AyobM. K., CheeK. L., HuangN. M., NeohH. M., LimH. N., Jamal R.andRahmanR. M. F. R. A. (2012). Nanoscale research letters 7(1):1-7.

45. Roy, I., RanaD., SarkarG., BhattacharyyaA., SahaN. R., MondalS., PattanayakS., ChattopadhyayS. and ChattopadhyayD. (2015). RSC Advances 5(32):25357-64.

46. Aswathi, R., AliM. M., ShuklaA.andSandhyaK. (2015). RSC Advances 5(40):32027-33. 